\title{
Aliskiren and Fenofibrate Constrict Liver Fibrosis by means of Focusing on TGF-ß1/Smad Signaling Pathway and Actuating HGF Expression
}

\author{
Yasmin Hashim', Nayira A. Abdel Baky², Mona M. Kamal², Amany M. Gad ${ }^{3,4 *}$ \\ ${ }^{1}$ Egyptian Ministry of Health and Population, Cairo, Egypt. \\ ${ }^{2}$ Department of Pharmacology and Toxicology, Faculty of Pharmacy (Girls), Al-Azhar University, Nasr City, Cairo, \\ Egypt. \\ ${ }^{3}$ Department of Pharmacology, Egyptian Drug Authority (EDA), Formerly (NODCAR), Giza, Egypt. \\ ${ }^{4}$ Department of Pharmacology and Toxicology, Faculty of Pharmacy, Sinai University (Kantara Branch), New City, El \\ Ismailia, Egypt. \\ * Correspondence: e-mail amany.gad@su.edu.eg_Tel: ++22-37244916
}

Article history: Received 19-12-2020 Revised 02-01-2021 Accepted 07-01-2021

Abstract: Aim of work: Liver fibrosis stems from changes in fibrotic genes expression in response to tissue damage in various chronic liver diseases, with no effective therapeutic program at present. The design of this study was to explore the possible protective effects and the molecular targets of aliskiren (ALS) and fenofibrate (FENO) against liver fibrosis which induced by carbon tetrachloride (CCL4) in rats. Methods: Wister albino rats had been injected with $0.4 \mathrm{ml} / \mathrm{kg} \mathrm{50 \%} \mathrm{CCL4,} \mathrm{three} \mathrm{times/week} \mathrm{for} \mathrm{eight} \mathrm{weeks,} \mathrm{to} \mathrm{establish} \mathrm{liver} \mathrm{fibrosis}$ model. Concurrently, ALS $(25 \mathrm{mg} / \mathrm{kg} /$ day $)$ and/or FENO $(25 \mathrm{mg} / \mathrm{kg} /$ day) were orally administrated for 8 weeks to rats CCL4 intoxicated. Results: Treatment with ALS and/or FENO ameliorated oxidative stress and hepatocellular damage in CCL4-intoxicated rats as indicated by the marked reduction in hepatic lipid peroxidation and serum transaminases with concomitant significant increase in hepatic superoxide dismutase (SOD) as well as reduced glutathione (GSH) content. The magnitude of liver inflammation and fibrosis was also alleviated by both medications, as is evident from the substantial decrease in hepatic proinflammatory and profibrotic cytokines namely tumor necrosis factor alpha (TNF-a), interlukin-6, (IL-6), C-reactive protein (CRP) and transforming growth factor- $\beta 1$ (TGF- $\beta 1$ ) with restrain in fibrous deposition alongside architecture alteration that was shown upon histopathological examination. Additionally, simultaneous administration of ALS with FENO downregulated hepatic p-Smad3 protein and increased hepatic growth factor (HGF) expression in CCL4-intoxicated rats. Conclusion: Conclusively, this study highlights the hepatoprotective effect of ALS and FENO and implies that their anti-fibrotic mechanism involves blockade of TGF- $\beta 1 / \mathrm{Smad}$ signaling pathway, induction of HGF expression, besides modulation of inflammation as well as oxidative stress.

Keywords: liver fibrosis; aliskiren; fenofibrate; TGF- $\beta$; p.samd3.

\section{INTRODUCTION}

Liver fibrosis is known as a reversible process of wound healing results from many pathological chronic liver diseases and continuous injury to the hepatic tissue ${ }^{1}$. It is distinguished by overproduction and accumulation of extracellular matrix (ECM), which damages the normal physiological structure of the liver ${ }^{2}$. Pathologically, in response to diverse types of chronic injurious insult, hepatocytes are damaged and diverse infiltrated immune cells activate normal quiescent vitamin A storing hepatic stellate (HSCs) cells to undergo trans-differentiation into collagen-producing myofibroblasts ${ }^{3}$. Overproduction of ECM result from persistent activation of these proliferating myofibroblasts,

Cite this article: Hashim, Y., Abdel Baky, N., Kamal, M., Gad, A. Aliskiren and Fenofibrate Constrict Liver Fibrosis by means of Focusing on TGF- $\beta 1 /$ Smad Signaling Pathway and Actuating HGF Expression. Azhar International Journal of Pharmaceutical and Medical Sciences, 2021; 1(1): 73-86 doi: 10.21608/aijpms.2021.54231.1028 
which end with the formation of scar tissue in fibrotic liver ${ }^{4-6}$. Many signal conduction pathways show a functional disorder in hepatic failure (HF), such as TGF- $\beta 1 /$ Smad, and nuclear factor $-\kappa B(N F-\kappa B)$ signaling ${ }^{7-9}$. Assumed these facts, it is a suitable approach to attenuate liver inflammation and inhibit TGF- $\beta 1 /$ Smad signaling for the treatment of liver fibrosis. Hepatocyte growth factor (HGF), a multifunctional protein that has arisen as a powerful anti-fibrotic cytokine and averts tissue fibrosis in numerous organs, including the liver ${ }^{10}$. It activates various biological processes, such as differentiation and morphogenesis through embryogenesis, organ regeneration, proliferation, survival, motility and tumour invasiveness ${ }^{11}$. Aliskiren is a treatment used for hypertension treatment; it reduces renin, which measured as the foremost-rate limiting enzyme in angiotensin II (Ang II) production and subsequently production of angiotensin I (Ang I) from angiotensinogen. Halting the renin action downregulates the Renin-Angiotensin-Aldosterone System (RAAS) ${ }^{12}$ and might be valuable by reducing oxidative stress and pro-inflammatory cytokines production in paracetamol induced hepatotoxicity ${ }^{13}$. Numerous studies showed that aliskiren reduces oxidative stress through inhibition of Ang II formation 14, 15. Moreover, it attenuates carbon tetrachloride-induced liver damage ${ }^{16}$. Aliskiren influences inflammatory cytokines 17,18 and oxidant/antioxidant balance ${ }^{18}$; thus, it is assumed to be utilized in cure of many diseases experimentally. Aliskiren may decrease Ang II production during the progression of chronic liver injury and alongside amend inflammation and fibrosis.

Fenofibrate is a valuable medication used for cure of atherogenic dyslipidemias, producing a significant decline in triglyceride and rise in cholesterol contents of high-density lipoprotein ${ }^{19,20}$. It exerts an antioxidant effect by reducing malondialdehyde and by stimulating the expression of SOD $^{21,22}$, its anti-inflammatory activity achieved by repressing the activity of transcription factors and by reducing plasma concentrations of TNF- $\alpha{ }^{22}$. Noteworthy, van der Veen 23 reported that administration of fenofibrate prohibited and somewhat reversed nonalcoholic fatty liver disease (NAFLD) in mice and reversed hepatic steatosis and fibrosis

Therefore, the present work is meant to inspect the possible anti-fibrotic properties of aliskiren and fenofibrate besides to elucidate their impact on the crucial events involved in liver fibrosis, concerning oxidative stress mechanism, inflammatory signals and TGF- $\beta 1 /$ Smad pathway.

\section{METHODS}

\subsection{Chemicals and Drugs}

Carbon tetrachloride (CCL4) had been purchased from Sigma-Aldrich for Chemical Co (St. Louis, MO, USA). ALS was purchased from Novartis Pharma (Basel, Switzerland). And fenofibrate had been purchased from Mepaco-MEDIFOOD (Cairo, Egypt).

\subsection{Animals}

Male Wister albino rats $(180 \pm 20 \mathrm{~g})$ had been obtained from (NRC), the National Institute for Research, Cairo, Egypt. The rats were kept at controlled environmental circumstances with relatively constant temperature at $\left(23 \pm 1^{\circ} \mathrm{C}\right)$, and adjusted humidity $(60 \pm 10 \%)$, as well as a $12 / 12 \mathrm{~h}$ light/dark cycle. They had been kept for 1 week before experiment for adaption and had been allowed standard rat chow and water ad libitum. The experimental protocol of the current study which applied had been approved from the Faculty of Pharmacy (Al-Azhar University), by the Animal Ethics Committee (No. 77 / 2016).

\subsection{Preparation of CCL4 for the establishment of} liver fibrosis model

CCL4 was dissolved using corn oil as a vehicle $(40 \% \mathrm{v} / \mathrm{v})$. The induction of liver fibrosis was done by intraperitoneal (i.p.) injection of $0.4 \mathrm{ml} / \mathrm{kg}$ of $\mathrm{CCL}_{4}$, three times, weekly for exactly 8 weeks.

\subsection{Experimental design}

To accomplish the goals of the current study, adult male albino rats (A total of 60) were randomly allocated into 6 groups of 10 animals:

Group 1(Control group): Rats were injected i.p. with normal saline daily $(0.9 \% \mathrm{NaCl})$ for 5-8 weeks and allotted as normal control group.

Group 2(Corn oil group): Rats were inoculated with corn oil only $(0.4 \mathrm{ml} / \mathrm{kg}$; i.p.; 3 times weekly) for 8 weeks.

Group 3 (CCL4 group): Rats were inoculated with CCL4 only $(0.4 \mathrm{ml} / \mathrm{kg}$; i.p.; 3 times weekly;24) for 8 weeks and allotted as positive control group.

Group 4 (CCL4+ALS group): Rats were inoculated i.p. with CCL4 $(0.4 \mathrm{ml} / \mathrm{kg}$; i.p.; 3 times weekly) and concomitantly treated with ALS 25 $\mathrm{mg} / \mathrm{kg} /$ day; (p.o.) for 8 weeks 25 . 
Group 5 (CCL4+FENO group): Rats were inoculated i.p. with CCL4 (0.4 ml $/ \mathrm{kg}$; i.p.; 3 times weekly) and concomitantly treated with FENO 25 $\mathrm{mg} / \mathrm{kg} /$ day; p. o. for 8 weeks ${ }^{26}$.

Group 6(CCL4+ALS+FENO group): Rats were inoculated i.p. with CCL4 $(0.4 \mathrm{ml} / \mathrm{kg}$; i.p.; 3 times weekly) and concomitantly treated with ALS and Feno, in the same regimen that was previously mentioned in group $4 \& 5$.

General conditions of the rats were observed daily throughout the whole experiment. Twenty-four hours after last dose administration of the treatment protocol, samples of blood were collected by retro-orbital sinus puncture under mild anesthesia, centrifuged at $(3,000 \mathrm{rpm})$ exactly for 15 minutes, and serum was isolated. The samples had been stored at $-80^{\circ} \mathrm{C}$ for analysis. Rats had been euthanized and cervical dislocation was applied then livers had been quickly and carefully separated, washed, and weighed.

\subsection{Assessment of liver enzymes and liver index}

Colorimetrical determination of aspartate aminotransferase (AST) as well as alanine aminotransferase (ALT), concentrations using specific enzyme-linked immunosorbent assay kits (MyBioSource; San Diego, USA) according to manufacturer's directions. Liver index was calculated according to the formula: (liver weight/body weight) $\times 100$.

\subsection{Assessment of inflammatory markers}

Liver tissues were washed and homogenized in ice-cold phosphate buffered saline (PBS) $(\mathrm{pH}=7.4)$ to obtain $10 \%$ homogenate $(\mathrm{w} / \mathrm{v})$, which was then centrifuged for $15 \mathrm{~min}$ at 5,000 rpm and $4{ }^{\circ} \mathrm{C}$. The supernatant obtained was used for measuring interleukin 6 (IL)-6, tumor necrosis factor alpha (TNF)- $\alpha$, C-reactive protein (CRP) using ELISA kit as per the manufacturer's instructions (R\&D Systems Inc., Minneapolis, MN, USA).

\subsection{Assessment of hepatic oxidative stress markers}

Concentration of malondialdehyde (MDA), which represent a lipid peroxidation index and oxidative stress, was measured by thiobarbituric acid (TBA) method ${ }^{27}$. The reaction is based on reaction of malondialdehyde with TBA at $98^{\circ} \mathrm{C}$. MDA was assessed by colorimetric analysis using assay kit (abcam, MA, USA) as said by manufacturer's instructions. GSH content of liver tissue was determined using commercially available kit supplied by Biodiagnostic, Giza, Egypt according to Beutler method ${ }^{28}$ method. Superoxide Dismutase was measured by commercial kits (Biodiagnostic, Cairo, Egypt) according to Nishikimi method ${ }^{29}$.

\subsection{Assessment of liver fibrosis markers}

ELISA kit was used to determine liver TGF- $\beta 1$ which supplied by MyBioSource (San Diego, USA). Further assessment of liver fibrosis was performed where, liver tissue of $\mathrm{p}$-SMAD3 protein and the expression of HGF were assessed by western blot analysis.

\subsection{Histopathological examination}

Livers were removed and instantly fixed in $10 \%$ neutral buffered formalin, followed by dehydration in ascending grades of ethanol, cleared in xylene and embedded in paraffin. $3 \mu \mathrm{m}$ thickness sections were cut and stained with haematoxylin and eosin $(\mathrm{H} \& \mathrm{E})$. In order to avoid bias, all histopathologic stages and assessment of specimens were done by a qualified observer blinded to the identity of the samples examined ${ }^{30}$.

\subsection{Statistical analysis}

The data analysis was conducted using statistical software SPSS (version 21). The data is expressed in the mean \pm S.D. and statistical analysis was performed using one way ANOVA accompanied by Bonferroni as a post-hoc measure was used for statistical significance at $\mathrm{p}<0.05$.

\section{RESULTS}

\subsection{Effect on Body Weight and Liver Weight in}

\section{Liver fibrosed Rats}

FENO and/or ALS lessen macroscopic changes in liver fibrosis induced by $\mathrm{CCL}_{4}$ in rats. At the end of treatment period, all rats of $\mathrm{CCL}_{4}$-treated groups observed weaker and lethargic. Nevertheless, rats in the combination-treated group, exhibited as much as slight of those symptoms. As revealed in table 1, treatment of rats with ALS and/or FENO did not significantly affect the body weight gain, as compared to normal control animals during the whole study. However, $\mathrm{CCL}_{4}$ - treated rats exhibited a significant rise in their liver weights compared to normal animals $(p<0.001)$. ALS and/or FENO treatment significantly suppressed this increase in 
liver weight compared to $\mathrm{CCL}_{4}$-treated rats. Additionally, two weeks following $\mathrm{CCL}_{4}$ treatment, $\mathrm{LW} / \mathrm{BW}$ ratio was increased compared to that of normal control group, even though, ALS or FENO treatment significantly reduced LW/BW in liver fibrosed rats. Interestingly, ALS and FENO treatment for 8 weeks restored the increase in $\mathrm{LW} / \mathrm{BW}$ ratio to control value (Table.1).

Table 1: Effects of Concurrent Treatment with Aliskiren, Fenofibrate, and their Combination on CCL4 -Induced Changes on Body Weight and Liver Weight in Liver fibrosed Rats. ( Data are presented as mean \pm S.D ( $n=6$ ). *P $<0.05$, compared to normal control group, $\pi \mathrm{P}<0.05$, compared to corn oil group; $\varphi \mathrm{P}<0.05$, compared to CCL4 group using ANOVA followed by Bonferroni as a post-ANOVA test)

\begin{tabular}{|c|c|c|c|c|}
\hline \multirow{2}{*}{ Groups } & \multicolumn{2}{|c|}{ Body Weight (g) } & \multirow{2}{*}{ liver weight (g) } & \multirow{2}{*}{ Liver Index } \\
\hline & Initial & Final & & \\
\hline Control & $173.8 \pm 22.58$ & $292.8 \pm 55.71$ & $10.09 \pm 1.428$ & $0.0325 \pm 0.0031$ \\
\hline $\begin{array}{c}\text { Corn } \\
\text { Oil } \\
\end{array}$ & $183.9 \pm 34.54$ & $283.9 \pm 53.55$ & $10.45 \pm 1.246$ & $0.0302 \pm 0.0017$ \\
\hline $\mathrm{CCl}_{4}$ & $187.2 \pm 27.05$ & $308.8 \pm 51.80$ & $12.54 \pm 1.139^{* \pi}$ & $0.0401 \pm 0.0029^{*} \pi \#$ \\
\hline $\begin{array}{c}\mathrm{CCl}_{4}+ \\
\mathrm{ALS}\end{array}$ & $194.4 \pm 30.90$ & $285.0 \pm 45.80$ & $11.78 \pm 1.024^{* \pi \varphi \# ~}$ & $0.0375 \pm 0.0044^{*} \pi$ \\
\hline $\begin{array}{l}\mathrm{CCl}_{4}+ \\
\text { FENO }\end{array}$ & $194.2 \pm 21.35$ & $310.6 \pm 29.65$ & $11.79 \pm 1.012^{*} \varphi \#$ & $0.0351 \pm 0.0033^{\varphi \# ~}$ \\
\hline $\mathrm{CCl}_{4}+$ & & & & \\
\hline $\begin{array}{l}\text { ALS+ } \\
\text { FENO }\end{array}$ & $193.5 \pm 19.58$ & $280.5 \pm 30.98$ & $11.47 \pm 1.047^{\varphi \#}$ & $0.0338 \pm 0.0031^{\varphi \#}$ \\
\hline
\end{tabular}

\subsection{Aliskiren and/or fenofibrate reduce the} production of liver injury biomarkers in CCL4-treated rats

In the current study, $\mathrm{CCL}_{4}$ treatment induced liver damage that was indicated by increased serum ALT and AST. Figure $1 \mathrm{~A}$ and $1 \mathrm{~B}$, respectively showed that, CCL4-treatment significantly increased ALT and AST concentrations by $79.58 \%$ and 70.97 $\%$, respectively compared with that of control rats $(\mathrm{p}<0.05)$. Nevertheless, concurrent treatment with ALS, FENO or their combination significantly lessened $(\mathrm{p}<0.05)$ ALT concentration by $47.21 \%$, $42.97 \% 74.53 \%$, and AST by $49.19 \%, 45.56$ $\%, 64.51 \%$, respectively compared to CCL4-treated rats. (Figure1A-B).
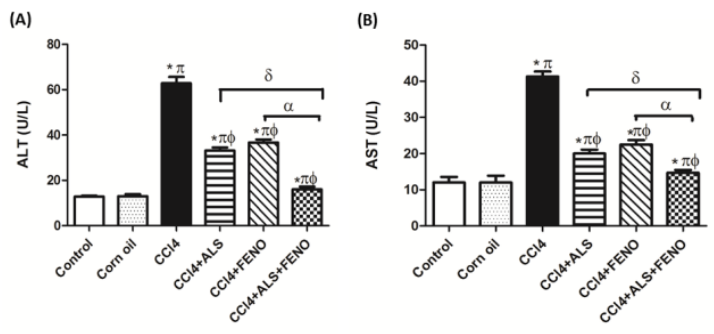

Figure 1(A-B): Effect of Aliskiren and/or fenofibrate on liver transaminases markers ; ALT (Panel A) and AST (Panel B) in CCL4-treated rats. Data are presented as mean \pm S.D $(\mathrm{n}=6) . * \mathrm{P}<0.05$, compared to normal control group, $\pi \mathrm{P}<0.05$, compared to corn oil , $\varphi \mathrm{P}<0.001$ compared to CCL4- liver fibrosed group, $\alpha \mathrm{P}<0.001$, compared to combination group, respectively, using ANOVA followed by Bonferroni as a post-ANOVA test.

\subsection{Aliskiren and/or fenofibrate reduce oxidative stress biomarkers production in $\mathrm{CCL}_{4}$ treated rats}

(Fig 2A-C) shows that, MDA was significantly heightened $(\mathrm{p}<0.05)$ in CCL4 treated animals by $68.45 \%$ as compared to control rats. ALS and/or FENO treatment significantly hampered $(\mathrm{p}<0.01)$ MDA by $49.57 \%, 53.62 \%$ and $72.24 \%$, respectively as compared to $\mathrm{CCL}_{4}$-treated group (Figure 2A). Also, hepatic GSH content in $\mathrm{CCL}_{4}$ treated group was significantly reduced by $65.13 \%$ $(\mathrm{p}<0.01)$. Treatment of $\mathrm{CCL}_{4}$ - treated animals with either ALS and/or FENO significantly increased hepatic GSH level by $54.85 \%, 53.57 \%$ and $60.28 \%$, respectively as compared to $\mathrm{CCL}_{4}$-treated group (Figure 2B). SOD level in $\mathrm{CCL}_{4}$ treated group was significantly reduced by $72.02 \%$ compared with normal group $(\mathrm{p}<0.01)$. Treatment of $\mathrm{CCL}_{4}$ - treated rats with either ALS and/or FENO significantly increased hepatic SOD level by $58.98 \%$, $60.25 \%$ and $68.67 \%$, respectively compared with that of $\mathrm{CCL}_{4}$-treated group (Figure 2C). 


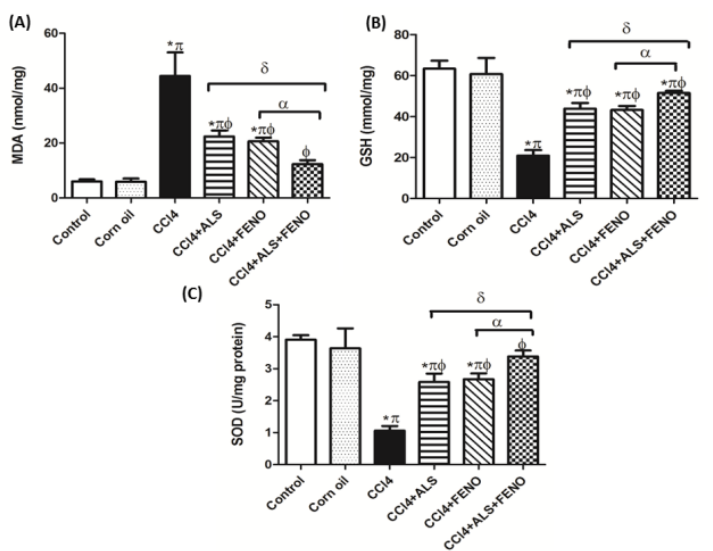

Figure 2(A-C): Aliskiren and/or fenofibrate reduce malondialdehyde level (MDA; Panel A), reduced glutathione content (GSH; Panel B) and superoxide dismutase activity (SOD; Panel C) in CCL4 treated rats. Data are presented as mean \pm S.D $(n=6) . * P<0.05$, compared to normal control group, $\pi \mathrm{P}<0.05$, compared to corn oil , $\varphi \mathrm{P}<0.001$ compared to CCL4- liver fibrosed group, $\alpha \mathrm{P}<0.001$, compared to combination group, respectively, using ANOVA followed by Bonferroni as a post-ANOVA test.

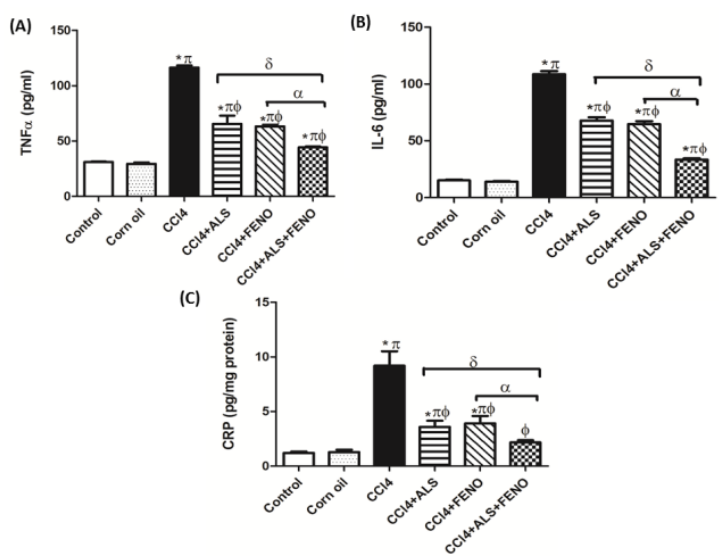

Figure 3(A-C): Aliskiren and/or fenofibrate reduce tumor necrosis factor alpha level (TNF- $\alpha$; Panel A), interleukin 6 level (IL-6; Panel B) and C-reactive protein (CRP; Panel C) in CCl4 treated rats. Data are presented as mean $\pm \mathrm{S} . \mathrm{D}(\mathrm{n}=6) . * \mathrm{P}<0.05$, compared to normal control group, $\pi \mathrm{P}<0.05$, compared to corn oil ,$\varphi \mathrm{P}<0.001$ compared to $\mathrm{CCl} 4-$ liver fibrosed group , $\alpha \mathrm{P}<0.001$, compared to combination group, respectively, using ANOVA followed by Bonferroni as a post-ANOVA test.

\subsection{Aliskiren and/or fenofibrate reduce}

\section{inflammatory markers production in $\mathrm{CCL}_{4}$ treated rats}

Additionally, there was a significant increase of inflammation in the liver of CCL4-treated rats as evidenced from the significant elevation $(\mathrm{p}<0.05)$ in hepatic IL-6, TNF- $\alpha$ and CRP by $86.02 \%, 73.32 \%$ and $86.85 \%$ when compared to control group. Though, administration of ALS, FENO and their combination reduced TNF- $\alpha$ level by $43.69 \%, 45.64$ $\%$ and $61.99 \%$ respectively when compared to $\mathrm{CCL}_{4}$ treated rats (Figure 3A), treatment with ALS, FENO and their combination decreased IL-6 level by $37.65 \%, 40.46 \%$ and $69.19 \%$, respectively as compared to $\mathrm{CCL}_{4}$ treated rats (Figure 3B) Treatment with ALS, FENO and their combination decreased CRP level by $61.61 \%, 59.05 \%$ and $78.26 \%$, respectively when compared to $\mathrm{CCL}_{4}$ treated rats. (Figure 3C).

\subsection{Aliskiren and/or fenofibrate reduce liver}

\section{fibrosis in $\mathrm{CCL}_{4}$-treated rats}

Figure 4 displayed that, rats treated with $\mathrm{CCL}_{4}$ exhibited a considerable rise $(p<0.05)$ in TGF- $\beta 1$ content by $50.93 \%$ compared with that of control rats. Nevertheless, treatment with ALS and/or FENO significantly reduced TGF- $\beta 1$ levels by $41.93 \%, 41.85 \%, 50.22 \%$, respectively compared to CCL4-treated rats $(\mathrm{p}<0.05)$. Interestingly, the combination regimen significantly decreased TGF- $\beta 1$ to a superior level $(p<0.05)$ than either ALS and/or FENO single treatment protocol.

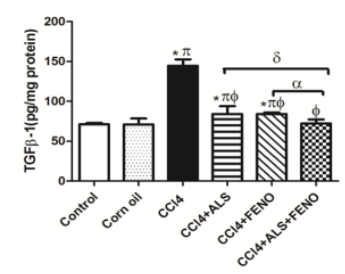

Figure (4): Effect of ALS and/or FENO Treatment on TGF- $\beta 1$ Level in Liver Fibrosed Rats. Data are presented as mean $\pm S . D(n=6) . * P<0.05$, compared to normal control group, $\pi \mathrm{P}<0.05$, compared to corn oil , $\varphi \mathrm{P}<0.001$ compared to CCL4 liver fibrosed group.

\subsection{Effect of Aliskiren and/or fenofibrate}

treatment on p-SMAD3, and hepatic growth

factor protein (HGF) levels in carbon

tetrachloride treated rats

Figure 5(A-B) shows western blotting of p-SMAD3, and HGF.CCL4-treatment significantly increased $(\mathrm{p}<0.05) \mathrm{p}$-SMAD3(5.3fold) in liver tissue compared with that of control rats. Though, treatment with ALS and/or FENO declined SMAD3 phosphorylation. Remarkably, treatment with ALS and FENO halted the levels of p-SMAD3 $(p<0.05)$ when compared with that of $\mathrm{CCL}_{4}$-treated 
animals (Figure 5A,). On the other hand, expression of HGF was significantly decreased after $\mathrm{CCL}_{4}$ treatment (18.17) compared to control group (Figure 5B). Treatment with ALS, FENO or their combination significantly increased HGF protein expression as compared with that of $\mathrm{CCL}_{4}$ treated rats. Remarkably, the ALS and FENO combination as a treatment significantly lessened SMAD3 phosphorylation, and markedly increased HGF expression to a higher extent $(\mathrm{p}<0.05)$ than either ALS or FENO treatment indicating a synergistic effect.

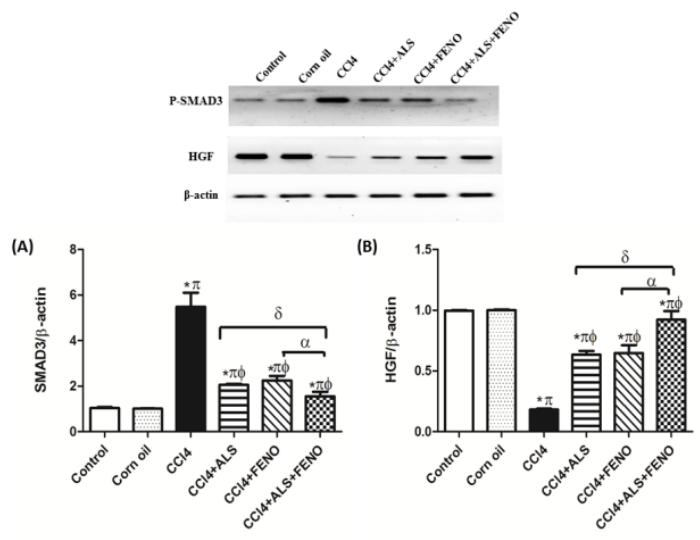

Figure 5(A-B): Effects of Concurrent Treatment with Aliskiren, Fenofibrate and their Combination on CCL4-Induced Changes on p-SMAD3 (Panel A), and HGF (Panel B) protein levels. Data are presented as mean \pm S.D $(\mathrm{n}=6) . * \mathrm{P}<0.05$, compared to normal control group, $\pi \mathrm{P}<0.05$, compared to corn oil , $\varphi \mathrm{P}<0.001$ compared to CCL4liver fibrosed group, $\alpha \mathrm{P}<0.001$, compared to combination group, respectively, using ANOVA followed by Bonferroni as a post-ANOVA test.

\subsection{Aliskiren and/or fenofibrate improve}

\section{histopathological architecture of liver tissue in $\mathrm{CCL}_{4}$ treated rats}

The tissue of these cells was stained with $\mathrm{H}$ and E staining (Figure 6). In $\mathrm{CCL}_{4}$ treated group, the histological sections of the liver showed congestion in portal vein, dilation of bile duct alongside inflammatory cell infiltration and oedema with fibrosis in the portal area as well as centrlobular vacuolization of hepatocytes also, nuclear pyknosis and cytomegaly in some of hepatocytes associated with fatty changes were observed (Figure 6C). The treatment with ALS or FENO showed mild hepatocyte vacuolization, fine fibrosis, and inflammatory cell infiltration (Figure 6D and Figure 6 E, respectively). However, treatment with combination of ALF and FENO showing hepatocyte fine fibrosis to a lesser extent (Figure 6F). The staining of collagen \& fibroblastic cells proliferation in hepatic tissue of different experimental groups using Masson's trichome staining (MT) showing negative MT reaction at central vein, portal area and surrounding parenchyma in liver of rats in control group \& corn oil group (Figure 7. A and Figure B, respectively) and showing severe positive reaction in portal area and extended to hepatic parenchyma forming lobules in CCL4 treated group (Figure 7C). The treatment with ALS or FENO showed mild to moderate positive reaction of MT (Figure $7 \mathrm{D}$ and Figure $7 \mathrm{E}$, respectively). However, treatment with combination of ALF and FENO negative reaction for MT (Figure 7 F).

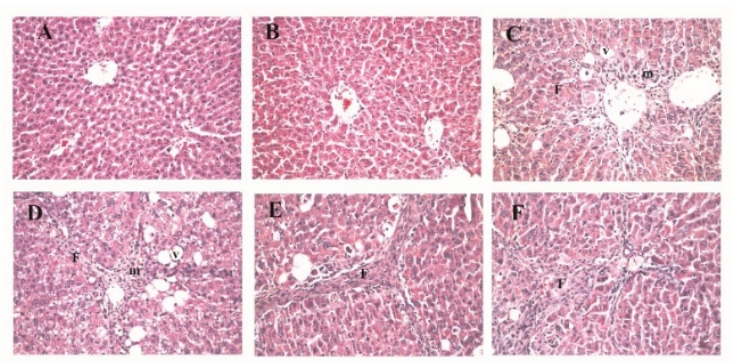

Figure (6): Representative photomicrographs of liver sections stained with H \& E (×40). A): Photomicrograph of liver section of control group normal hepatic architecture, hepatocyte structure and central vein $(\mathrm{CV})$. (B): Photomicrograph of liver section of corn oil group showing no histopathological alterations. (C): Photomicrograph of liver section of $\mathrm{CCL}_{4}$ group showing inflammatory cells infiltration $(\mathrm{m})$, fibroblastic cells proliferation in the portal triad $(\mathrm{F})$, nuclear pyknosis and vacuolization (v) in some hepatocytes associated with fatty changes. (D): Photomicrograph of liver section of ALS co-treated group showing mild hepatocyte vacuolization, fine fibrosis, and inflammatory cell infiltration. (E): Photomicrograph of liver section of fenofibrate co-treated group showing mild hepatocyte fine fibrosis, and inflammatory cell infiltration. (F): Photomicrograph of liver section of combination showing mild hepatocyte fine fibrosis.

\section{DISCUSSION}

Liver fibrosis happens as a common obsessive result of constant liver wounds of distinctive cause $^{31}$. It speaks to a major wellbeing care burden with increment in frequency around the world ${ }^{32}$. Developing exploratory and clinical confirmations are demonstrating that liver fibrosis is reversible ${ }^{33}$, in this way worth to be a point of investigation all inclusive. For numerous a long time, the special regenerative capacities of liver have been a profoundly motivating point of inquiring about. 


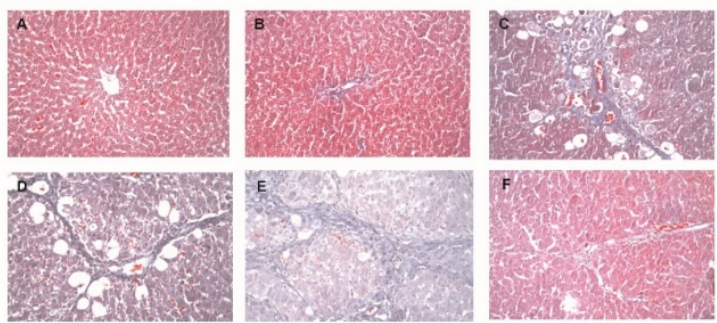

Figure (7): Representative photomicrographs of liver sections stained with Masson's trichrome $(\times 40)$.A): Photomicrograph of liver section of control group showing no collagen fibers. (B): Photomicrograph of liver section of corn oil group showing no collagen fibers. (C): Photomicrograph of liver section of $\mathrm{CCL}_{4}$ group showing heavy collagen fibers deposition and pseudolobules formation (bridging fibrosis). (D): Photomicrograph of liver section of Aliskiren co-treated group showing complete absence of collagen fibers. (E): Photomicrograph of liver section of fenofibrate co-treated group showing no-collagen fibers. (F): Photomicrograph of liver section of ALS and FENO combination co-treated group showing no collagen fibers.

Knowing this exceptional preparation may open components of hepatic regenerative capacities and aid the field of regenerative pharmaceutical to move forward considerably ${ }^{34}$. CCL4 is broadly utilized tentatively to consider liver aggravation, fibrosis and cirrhosis ${ }^{35}$. It actuates hepatic harm by the arrangement of free radicals amid reductive dehalogenation by cytochrome P-450 amid its digestion system which causes lipid peroxidation of cellular films, driving to corruption. The initial events of CCL4 evoke the secondary mechanisms which ultimately disrupt the plasma membrane and cause cell death ${ }^{36}, 37$. The purpose of this experiment is to study the antifibrotic potential of ALS and FENO alone or in combination on liver fibrosis induced by CCL4 in rats. This experiment showed that the combination of ALS and FENO had significantly higher antifibrotic effect than each one alone. There was significant decline of hydroxyproline level, fibrotic area, inflammatory markers, and oxidative stress in the combination group compared with each one alone. CCL4 produced marked liver damage, this is evidenced by enzymatic level alterations in our study, which include risen in the levels of serum ALT and AST, which are considered as markers of cellular leak, functional integrity loss of the cell membrane, and decline of metabolic capacity in liver tissue. These findings are consistent with those of previous studies ${ }^{38-40}$. The elevation in serum hepatic enzymes indicated deterioration in hepatic function due to parenchymal injury after CCL4 administration. Treatment with ALS or FENO compared with the model control group, the contents of ALT and AST were significantly reduced. The abovementioned biochemical aberrations were more long-established by the examination of histology of liver tissue which exposed massive hepatocellular deterioration in the CCL4 treated rats, that lessened by ALS or FENO co-treatment. These results showed the probable hepatoprotective action of ALS or FENO. Our findings agree with previous studies reporting the capability of ALS or FENO to protect against alcohol, carbon tetrachloride and steatohepatitis induced hepatic tissue damage ${ }^{41-43}$. One of the major markers of hepatic harm is oxidative stress-mediated lipid peroxidation which plays irreversible harm to hepatocellular components. MDA is the item of lipid peroxidation in cell films and harms the judgment of cells. It too causes a lopsidedness to lysosomes and makes a difference to oxidize the protein ${ }^{44}$. Lipid peroxidation is one of the major viewpoints of CCL4- inebriated liver harm ${ }^{45},{ }^{46}$ and is interceded by the free radical subordinates of CCL4. The hepatic harm was conceivably related with at slightest two successive forms the starting stage included CYP 450-mediated bioactivation of CCL4 to an exceedingly receptive trichloromethyl radical and ROS, driving to lipid peroxidation and harm of hepatocyte layer. This was taken after the discharge of a development variables fiery arbiters and prostaglandins from enacted hepatic macrophages, which potentiate CCL4 -induced hepatic harm ${ }^{47}$. Also, in our study CCL4 -induced a significant decline in the constitutive GSH level and SOD activity which adversely affects cellular thiol redox balance as well as cellular defense system against superoxide radicals and potentially made the cells susceptible to a several internal and environmental stress ${ }^{48}$. Decrease in GSH level and SOD activity might be due to the increased utilization of the hepatocytes in scavenging toxic radical of CCL4 ${ }^{49}$. We found that, treatment with aliskiren or fenofibrate strongly guarded against CCL4-induced oxidative stress via preservation of lipid peroxidation and restoration of the hepatic antioxidant defense system. Together with our results, previous studies have reported potent antioxidant activity of aliskiren ${ }^{50}$ and fenofibrate ${ }^{41}$. Rashikh ${ }^{14}$ reported that, aliskiren preserved the antioxidant defense and increased some antioxidant markers like glutathione (GSH) and superoxide dismutase (SOD) in a model of cardiomyopathy in rats. RAAS activation and enhanced Ang II activity exacerbate oxidative stress 51. Inhibition of the RAAS by aliskiren together with decreased Ang II levels reduce the oxidative stress that causes tissue damage ${ }^{14}$. Earlier studies 
performed with captopril and aliskiren showed that inhibition of Ang II reduced the production of free oxygen radicals ${ }^{18}$, which is consistent with our results. Therefore, the suppression of the RAAS with aliskiren in the CCL4-induced fibrosis could reduce the oxidative stress-induced damage. Also, as presented here, the improvement of hepatic fibrosis by fenofibrate administration could be due to a reduction in reactive oxygen species-induced activation of hepatic stellate cells ${ }^{23}$, where PPAR $\alpha$ directly stimulates catalase expression ${ }^{52}$. Without a doubt, irritation is considered as one of the critical components by which CCL4 intercedes liver fibrosis ${ }^{53}$. Numerous ponders, in agreement with our comes about, appeared that CCL4 increments the hepatic TNF- $\alpha$ level ${ }^{54,55}$. In expansion Kurt 56 expressed that, CCL4 causes intense harm to lung tissue and leads to cytokine generation, oxidative stretch, and apoptosis. CCL4 leads to tissue harm in different tissues by expanding TNF- $\alpha$ and NO generation ${ }^{57}$. Too, CCL4 heightened the level of IL-6 and CRP. These findings agree with previous studies $^{23}$. Contrarily, It was already detailed that aliskiren neutralized paracetamol Induced actuated discharge of distinctive cytokines ${ }^{13}$. Angiotensin II is a strong inducer of oxidative stress where it increases oxidative stress and inflammation ${ }^{58}$.

In this study, aliskiren administration decreased TNF- $\alpha$, IL- 6 , and CRP that might be owing to the inhibition of Ang II, or due to antioxidant or anti-inflammatory properties of aliskiren $^{59}$. Aliskiren, in a dose-dependent design, altogether diminished aggravation and diminished serum concentrations of TNF- $\alpha$, CRP in rat models of irritation ${ }^{60}$. Comes about may well be ascribed to the capacity of aliskiren to diminish the concentration of incendiary variables ${ }^{61}$. Within the same setting, in this think about organization of fenofibrate hampered the rise of TNF- $\alpha$, IL- 6 and CRP levels. Interests, fenofibrate may progress affront affectability by constraining lipid amassing in a few tissues, counting the liver and muscles ${ }^{62},{ }^{63}$. This might be ascribed to augmented adiponectin collected with reduced IL- 6 and TNF- $\alpha$, CRP as reported TNF- $\alpha$ hepatic expression is reduced by PPAR $\alpha$ activation ${ }^{64}$.

Such an effect of fenofibrate on TNF $\alpha$, IL-6 and CRP is also in agreement with another report ${ }^{65}$. In well-designed studies, fibrate markedly reduced CRP levels in several studies through its anti-inflammatory effect ${ }^{66-68}$. Experimental studies proposed that fenofibrate had a protective role in prevention of Non-alcoholic fatty liver disease (NAFLD), provided that descriptive mechanisms where fenofibrate prohibited the accumulation of hepatic TG in an experimental model induced by high fat diet ${ }^{69,70}$. Subsequently, all histological findings of NAFLD were reversed. In the present study, chronic CCL4 administration activated TGF- $\beta$ /SMAD signaling pathway which is evidenced by significant elevated tissue level of TGF- $\beta$ as well as overexpression of nuclear p.samd 3 and significantly decrease in HGF expression. These findings are alike to those from previous studies of CCL4 effects in liver tissue ${ }^{71-73}$. SMAD 2 and SMAD 3 interact together to mediate TGF- $\beta$ signal transduction but only SMAD 3 appears to be key element responsible for fibrosis ${ }^{75}$. A several fibrogenic markers as collagen and alpha smooth muscle actin ( $\alpha$-SMA) are found to be SMAD 3 dependent ${ }^{76}$. HGF expression is related to changing development factor- $\beta$ (TGF- $\beta$ ) level because it appeared that the TGF- $\beta$ showed up to hinder HGF amalgamation through concealment of HGF gene expression ${ }^{77}$. Within the same setting, Li ${ }^{78}$, the higher the proportion of TGF- $\beta 1 / \mathrm{HGF}$ come about in more prominent expression of collagen and $\alpha$-SMA in unremitting rhinosinusitis patients and concluded that HGF can antagonize the fibrotic impact caused by TGF- $\beta 1$. Eminently, ALS or FENO co-treatment altogether restrained TGF- $\beta /$ SMAD signaling pathway as demonstrated by critical diminish in TGF- $\beta 1$ levels and noteworthy expression of p.samd3 related with critical increment in HGF expression. Past thinks about were in reliable with our comes about. On one hand, aliskiren or fenofibrate appeared enhancement in gentamicin-induced renal tubular cell apoptosis and fibrosis demonstrate through TGF- $\beta /$ SMAD subordinate way ${ }^{79}$. Additionally, the increment of HGF expression, could be auxiliary to Ang II arrangement restraint and extinguishing TGF- $\beta$ of by aliskiren. Ang II bar essentially expanded renal HGF mRNA and avoided renal harm in suddenly hypertensive rats $^{77}$. And on the other hand, a self-evident diminishment of TGF- $\beta 1$ levels and noteworthy moo expression of $\mathrm{p}$. samd 3 related with critical increment in HGF expression in fenofibrate bunch. These discoveries are reliable with Chanda ${ }^{80}$ and Al-Rasheed ${ }^{81}$. Another study, $\mathrm{Chen}^{82}$, also affirmed that fenofibrate reduced TGF- $\beta 1$ expression which in the long run comes about in less extracellular lattice statement. Additionally, the increment of HGF expression, could be auxiliary to Ang II arrangement restraint and extinguishing TGF- $\beta$ of by aliskiren. Ang II bar essentially expanded renal HGF mRNA and avoided renal harm in suddenly hypertensive rats ${ }^{83}$, In fibroblast cell of lung line of a human fetal, TGF- $\beta 1$ was demonstrated to drastically down-regulate HGF gene expression ${ }^{84}$. 


\section{CONCLUSION}

In conclusion, the research confirmed an evidence that aliskiren with fenofibrate could have a hepatoprotective and antifibrotic properties, effects which could be through lessening oxidative stress, declining the consequent inflammatory cascade along with hindering the pathway namely; TGF- $\beta /$ SMAD signaling. These data obviously showed the synergistic effect of aliskiren with fenofibrate, which might signify a novel therapeutic approach for treating hepatic fibrosis.

\section{Acknowledgments}

The authors would like to acknowledge Prof. Dr. A. Bakear (Pathology Department, Faculty of Veterinary Medicine, Cairo University, Cairo, Egypt) for his aid in the histopathological estimations.

\section{Conflicts of interest}

The authors report no conflict of interest.

Ethical statement: All procedures performed in this study were conducted in accordance with the regulations approved by the Ethics Committee at Faculty of Pharmacy, Al-Azhar University (permit number: (No. 77 / 2016).

\section{Author contribution}

YA, MK, and NAA conceived and designed research. YA, MK, AMG, and NAA conducted experiments. YA, MK, AMG and NAA analyzed data. YA, MK, AMG and NAA wrote the manuscript. All authors read and approved the final manuscript. All data were generated at Faculty of Pharmacy (Girls), Al-Azhar University, Nasr City, Cairo, Egypt.

\section{Funding}

This research did not receive any fund

\section{List of abbreviations:}

ALS; aliskiren,

Ang I; angiotensin I,

Ang II; angiotensin II,

CCL4; carbon tetrachloride,

CRP; C-reactive protein,

ECM; extracellular matrix,

FENO; fenofibrate,

GSH; reduced glutathione,

HF; hepatic failure,

HGF; Hepatocyte growth factor,

HSCs; hepatic stellate cells, i.p.; intraperitoneal,

IL-6; interlukin-6,

NAFLD; nonalcoholic fatty liver disease,

RAAS; Renin-Angiotensin-Aldosterone System,

SOD; superoxide dismutase,

TGF- $\beta 1$; transforming growth factor- $\beta 1$,

TNF-a; tumor necrosis factor alpha,

$\alpha$-SMA; Alpha smooth muscle actin

\section{REFERENCES}

1. Elfeky MG, Mantawy EM, Gad AM, Fawzy HM, El-Demerdash E. Mechanistic aspects of antifibrotic effects of honokiol in Con A-induced liver fibrosis in rats: Emphasis on TGF- $\beta$ /SMAD/MAPK signaling pathways. Life Sciences.2020;240:11709 DOI: $\underline{10.1016 / j .1 f s .2019 .117096 .}$

2. Balta $\mathrm{C}$, Ciceu A, Herman $\mathrm{H}$, Rosu M, Boldura OM, Hermenean A. Dose-dependent antifibrotic effect of chrysin on regression of liver fibrosis: the role in extracellular matrix remodeling. Dose-Response. 2018;16(3):155 doi.org/10.1177/1559325818789835

3. Lepreux S, Desmoulière A. Human liver myofibroblasts during development and diseases with a focus on portal (myo) fibroblasts. Frontiers in physiology. 2015;6:173

DOI: $\underline{10.3389 / \text { fphys.2015.00173 }}$

4. Puche JE, Saiman Y, Friedman SL. Hepatic stellate cells and liver fibrosis. Comprehensive Physiology. 2011;3(4):1473-92.

5. Wick G, Grundtman C, Mayerl C, Wimpissinger T-F, Feichtinger J, Zelger $\mathrm{B}$, et al. The immunology of fibrosis. Annual review of immunology. 2013;31:107-35.

6. Parola M, Pinzani M. Liver fibrosis: Pathophysiology, pathogenetic targets and clinical issues. Molecular aspects of medicine. 2019;65:37-55.

7. Li W, Zhu C, Chen X, Li Y, Gao R, Wu Q. Pokeweed antiviral protein down-regulates $\mathrm{Wnt} / \beta$-catenin signalling to attenuate liver fibrogenesis in vitro and in vivo. Digestive and Liver Disease. 2011;43(7):559-66.

8. Inokuchi S, Aoyama $\mathrm{T}$, Miura $\mathrm{K}$, Österreicher CH, Kodama Y, Miyai K, et 
al. Disruption of TAK1 in hepatocytes causes hepatic injury, inflammation, fibrosis, and carcinogenesis. Proceedings of the National Academy of Sciences. 2010;107(2):844-9.

9. Hu Z, Qin F, Gao S, Zhen Y, Huang D, Dong L. Paeoniflorin exerts protective effect on radiation-induced hepatic fibrosis in rats via TGF- $\beta 1 /$ Smads signaling pathway. American journal of translational research. 2018;10(3):1012 PMCID: PMC5883141

10. Xia J-L, Dai C, Michalopoulos GK, Liu Y. Hepatocyte growth factor attenuates liver fibrosis induced by bile duct ligation. The American journal of pathology. 2006;168(5):1500-12.

11. Gallo S, Sala V, Gatti S, Crepaldi T. Cellular and molecular mechanisms of $\mathrm{HGF} / \mathrm{Met}$ in the cardiovascular system. Clinical science. 2015;129(12):1173-93.

12. Buczko W, Hermanowicz JM. -Pharmacokinetics and pharmacodynamics of aliskiren, an oral direct renin inhibitor. Pharmacological reports. 2008;60(5):623

PMID: 19066408 Review

13. Karcioglu SS, Palabiyik SS, Bayir Y, Karakus E ,Mercantepe T, Halici Z, et al. The role of RAAS inhibition by aliskiren on paracetamol-induced hepatotoxicity model in rats. Journal of cellular biochemistry. 2016;117(3):638-46.

14. Rashikh A, Ahmad SJ, Pillai KK, Kohli K, Najmi AK. Aliskiren attenuates myocardial apoptosis and oxidative stress in chronic murine model of cardiomyopathy. Biomedicine \& Pharmacotherapy. 2012;66(2):138-43.

15. Wang Z, Liu Y, Han Y, Guan W, Kou X, $\mathrm{Fu}$ J, et al. Protective effects of aliskiren on ischemia-reperfusion-induced renal injury in rats. European journal of pharmacology. 2013;718(1-3):160-6.

16. Lee $\mathrm{KC}$, Chan CC, Yang YY, Hsieh YC, Huang YH, Lin HC. Aliskiren attenuates chronic carbon tetrachloride-induced liver injury in mice. European journal of clinical investigation. 2012;42(12):1261-71.

17. Aihara Y, Yoshiji H, Noguchi R, Kaji K, Namisaki T, Shirai Y, et al. Direct renin inhibitor, aliskiren, attenuates the progression of non-alcoholic steatohepatitis in the rat model. Hepatology research. 2013;43(11):1241-50.
18. Ziypak T, Halici Z, Alkan E, Akpinar E, Polat B, Adanur S, et al. Renoprotective effect of aliskiren on renal ischemia/reperfusion injury in rats: electron microscopy and molecular study. Renal Failure. 2015;37(2):343-54.

19. Melenovsky V, Stulc T, Kozich V, Grauova B, Krijt J, Wichterle D, et al. Effect of folic acid on fenofibrate-induced elevation of homocysteine and cysteine. American heart journal. 2003;146(1):110A-5A.

20. Legendre C, Caussé E, Chaput E, Salvayre $\mathrm{R}$, Pineau $\mathrm{T}$, Edgar AD. Fenofibrate induces a selective increase of protein-bound homocysteine in rodents: a PPAR $\alpha$-mediated effect. Biochemical and biophysical research communications. 2002;295(5):1052-6.

21. Yang T-L, Chen M-F, Luo B-L, Yu J, Jiang J-L, Li Y-J. Effect of fenofibrate on LDL-induced endothelial dysfunction in rats. Naunyn-Schmiedeberg's archives of pharmacology. 2004;370(2):79-83.

22. Li S, Gokden N, Okusa MD, Bhatt R, Portilla D. Anti-inflammatory effect of fibrate protects from cisplatin-induced ARF. American Journal of Physiology-Renal Physiology. 2005; 289(2): F469-F480.

23. van der Veen JN, Lingrell S, Gao $X$, Takawale A, Kassiri Z, Vance DE, et al. Fenofibrate, but not ezetimibe, prevents fatty liver disease in mice lacking phosphatidylethanolamine

$\mathrm{N}$-methyltransferase. Journal of lipid research. 2017;58(4):656-67.

24. Attia H, Al-Rasheed N, Mohamad R, Al-Rasheed N, Al-Amin M. The antifibrotic and fibrolytic properties of date fruit extract via modulation of genotoxicity, tissue-inhibitor of metalloproteinases and nuclear factor-kappa B pathway in a rat model of hepatotoxicity. BMC complementary and alternative medicine. 2016;16(1):414 DOI: $\underline{10.1186 / \mathrm{s} 12906-016-1388-2}$

25. Hussain SA, Utba RM, Assumaidaee AM. Effects of azilsartan, aliskiren or their combination on high fat diet-induced non-alcoholic liver disease model in rats. Medical Archives. 2017;71(4):251DOI: 10.5455/medarh.201 7.71.251-255

26. Rivera-Meza M, Muñoz D, Jerez E, Quintanilla ME, Salinas-Luypaert C, Fernandez K, et al. Fenofibrate 
administration reduces alcohol and saccharin intake in rats: possible effects at peripheral and central levels. Frontiers in Behavioral Neuroscience. 2017;11:133 DOI: 10.3389/fnbeh.2017.00133.

27. Kei S. Serum lipid peroxide in cerebrovascular disorders determined by a new colorimetric method. Clinica chimica acta. 1978;90(1):37-43.

28. Beutler E. Improved method for determination of blood glutathione. J Lab Clin Med. 1963;61(5):882-8.

29. Nishikimi M, Rao NA, Yagi K. The occurrence of superoxide anion in the reaction of reduced phenazine methosulfate and molecular oxygen. Biochemical and biophysical research communications. 1972;46(2):849-854. DOI: $10.1016 / \mathrm{s} 0006-291 \times(72) 80218-3$

30. Bancroft, J. D., \& Gamble, M. (Eds.). (2008). Theory and practice of histological techniques. Elsevier health sciences. pp. 129. Edinburgh: Churchill-Livingstone

31. Friedman SL. Liver fibrosis-from bench to bedside. Journal of hepatology. 2003;38:38-53.

32. Henderson NC, Iredale JP. Liver fibrosis: cellular mechanisms of progression and resolution. Clinical Science. 2007;112(5):265-80.

33. Ismail MH, Pinzani M .Reversal of hepatic fibrosis: pathophysiological basis of antifibrotic therapies. Hepatic medicine: evidence and research. 2011;3:69 DOI: 10.2147/HMER.S9051.

34. Gilgenkrantz H, de l'Hortet AC. Understanding liver regeneration: from mechanisms to regenerative medicine. The American journal of pathology. 2018;188(6):1316-27.

35. Jeong D-H, Lee G-P, Jeong W-I, Do S-H, Yang H-J, Yuan D-W, et al. Alterations of mast cells and TGF- $\beta 1$ on the silymarin treatment for CCL4-induced hepatic fibrosis. World journal of gastroenterology: WJG. 2005;11(8):1141 DOI: $10.3748 / w j g . v 11.18 .1141$

36. Recknagel RO, Glende Jr EA, Dolak JA, Waller RL. Mechanisms of carbon tetrachloride toxicity. Pharmacology \& therapeutics. 1989;43(1):139-54.

37. Eidi A, Mortazavi P, Bazargan M, Zaringhalam J. Hepatoprotective activity of cinnamon ethanolic extract against $\mathrm{CCI}_{4}$-induced liver injury in rats. Excli
Journal.

PMCID: PMC4990741.

38. Lu Y-H, Tian C-R, Gao C-Y, Wang W-J, Yang W-Y, Kong X, et al. Protective effect of free phenolics from Lycopus lucidus Turcz. root on carbon tetrachloride-induced liver injury in vivo and in vitro. Food \& nutrition research. 2018;62DOI: 10.29219/fnr.v62.1398.

39. Truong NH, Nguyen NH, Le TV, Vu NB, Huynh N, Nguyen TV, et al. Comparison of the treatment efficiency of bone marrow-derived mesenchymal stem cell transplantation via tail and portal veins in CCL4-induced mouse liver fibrosis. Stem cells international. 2016;2016: 13 doi.org/10.1155/2016/5720413.

40. Alam MA, Sagor AT, Tabassum N, Ulla A, Shill MC, Rahman GMS, et al. Caffeic acid rich Citrus macroptera peel powder supplementation prevented oxidative stress, fibrosis and hepatic damage in $\mathrm{CCL}_{4}$ treated rats. Clinical Phytoscience. 2018;4(1):14 https://doi.org/10.1186/s40816-018-0074y.

41. Xie C, Li L, Xu Y, Zhu Y, Jiang J. Anti-fibrosis effects of fenofibrate in mice with hepatic fibrosis. Zhonghua ganzangbing zazhi Chinese journal of hepatology. 2013;21(12):914-9. doi: 10.3760/cma.j.issn.1007-3418.2013.12.00 8. PMID: 24636293.

42. Kostapanos MS, Kei A, Elisaf MS. Current role of fenofibrate in the prevention and management of non-alcoholic fatty liver disease. World Journal of Hepatology. 2013;5(9):470 doi: 10.4254/wjh.v5.i9.470. PMID: 24073298; PMCID: PMC3782684.

43. El-Kholi E, El-beltagi HM, Abdo VB. Evaluation of the possible antifibrotic effect of aliskiren, valsartan, chloroquine, zafirlukast and colchicine on liver fibrosis induced by carbon tetrachloride in rats. $\mathrm{Br}$ J Med Health Res. 2015;2(7):41-54..

44. Gressner OA, Weiskirchen R, Gressner AM. Biomarkers of liver fibrosis: clinical translation of molecular pathogenesis or based on liver-dependent malfunction tests. Clinica Chimica Acta. 2007;381(2):107-13.

45. Basu S. Carbon tetrachloride-induced lipid peroxidation: eicosanoid formation and their regulation by antioxidant nutrients. Toxicology. 2003;189(1-2):113-27. 
46. Tripathi A, Yadav AK, Mishra PK, Rao CV. Hepatoprotective activity of ficus carica linn stems extract against carbon tetrachloride liver damage in wistar rats. 2017; 6 (5): 766-778.

47. Sudo K, Yamada Y, Moriwaki H, Saito K, Seishima M. Lack of tumor necrosis factor receptor type 1 inhibits liver fibrosis induced by carbon tetrachloride in mice. Cytokine. 2005;29(5):236-44.

48. Sirag H, Ibrahim H, Amer A, Salam T, Samaka R, El-Mowafy Y. Ameliorative effect of caffeic acid phenethyl ester and candsartain cilexetil on CCL4 induced hepatotoxicity in male rats. Annals of Biological Research. 2011;2(4):503-15.

49. Hassan S, Elbakry M, Mangoura S, Omar $Z$. Evaluation of the effect of Gallic acid against carbon tetrachloride-induced liver injury in albino rats: histological, immunohistochemical and biochemical study. Journal of Medical Histology. 2017;1(2):202-15.

50. Kamal S. Aliskiren Augments the Activities of Anti-Oxidant Enzymes in Liver Homogenates of DOCA Salt-Induced Hypertensive Rats. Advances in Enzyme Research. 2014;2014 DOI: 10.4236/aer.2014.22010.

51. Landmesser U, Spiekermann S, Preuss C, Sorrentino S, Fischer D, Manes C, et al. Angiotensin II induces endothelial xanthine oxidase activation: role for endothelial dysfunction in patients with coronary disease. Arteriosclerosis, Thrombosis, and Vascular Biology. 2007;27(4):943-8.

52. Toyama $\mathrm{T}$, Nakamura $\mathrm{H}$, Harano $\mathrm{Y}$, Yamauchi N, Morita A, Kirishima T, et al. PPAR $\alpha$ ligands activate antioxidant enzymes and suppress hepatic fibrosis in rats. Biochemical and biophysical research communications. 2004;324(2):697-704.

53. Wang R, Wang J, Song F, Li S, Yuan Y. Tanshinol ameliorates CCL4-induced liver fibrosis in rats through the regulation of $\mathrm{Nrf} 2 / \mathrm{HO}-1$ and $\mathrm{NF}-\kappa \mathrm{B} / \mathrm{I} \kappa \mathrm{B} \alpha$ signaling pathway. Drug design, development and therapy. 2018;12:1281-1292 doi: $10.2147 / D D D T . S 159546$

54. Zhang J, Yang A, Wu Y, Guan W, Xiong $\mathrm{B}$, Peng $\mathrm{X}$, et al. Stachydrine ameliorates carbon tetrachloride-induced hepatic fibrosis by inhibiting inflammation, oxidative stress and regulating MMPs/TIMPs system in rats. Biomedicine \& Pharmacotherapy. 2018;97:1586-94.
55. Toriumi K, Horikoshi Y, Osamura RY, Yamamoto Y, Nakamura N, Takekoshi S. Carbon tetrachloride-induced hepatic injury through formation of oxidized diacylglycerol and activation of the $\mathrm{PKC} / \mathrm{NF}-\kappa \quad \mathrm{B}$ pathway. Laboratory Investigation. 2013;93(2):218-29.

56. Kurt A, Tumkaya L, Yuce S, Turut H, Cure MC, Sehitoglu I, et al. The protective effect of infliximab against carbon tetrachloride-induced acute lung injury. Iranian journal of basic medical sciences. 2016;19(6):685-691.

57. Chen Z-Z, Wang Z-L, Deng C-Y, Zheng $\mathrm{H}$, Wang $\mathrm{X}-\mathrm{H}$, Ma L, et al. (Z)-5-(4-methoxybenzylidene)

thiazolidine-2, 4-dione protects rats from carbon tetrachloride-induced liver injury and fibrogenesis. World journal of gastroenterology: WJG. 2012;18(7):654-661.

58. Kim S-M, Kim Y-G, Jeong K-H, Lee S-H, Lee T-W, Ihm C-G, et al. Angiotensin II-induced mitochondrial Nox4 is a major endogenous source of oxidative stress in kidney tubular cells. PloS one. 2012;7(7):e39739

DOI: 10.1371/journal.pone.0039739

59. Al-Aubaidy HA, Sahib HA, Mohammad BI, Hadi NR, Abas SM. Antiatherosclerotic potential of aliskiren: its antioxidant and anti-inflammatory effects in rabbits: a randomized controlled trial. Journal of Pharmaceutical Technology and Drug Research. 2013;2(1):11 doi: 10.7243/2050-120X-2-11.

60. Aziz TA, Kareem AA, Othman $\mathrm{HH}$, Ahmed ZA. The Anti-Inflammatory Effect of Different Doses of Aliskiren in Rat Models of Inflammation. Drug Design, Development and Therapy. 2020;14:2841-51.

61. Karakousis K, Pinakas V, Kontovos L, Gavra M, Pinaka O. Pleiotropic effect of aliskiren in reducing levels of hscrp and fibrinogen when added as treatment in patients previously in treatment with valsartan and amlodipine plus valsartan: pp. 24.484. Journal of Hypertension. 2010;28:e391 doi: 10.1097/01.hjh.0000379410.77854.c9.

62. Buldak L, Dulawa-Buldak A, Labuzek K, Okopien B. Effects of 90-day hypolipidemic treatment on insulin resistance, adipokines and proinflammatory cytokines in patients 
with mixed hyperlipidemia and impaired fasting glucose. International journal of clinical pharmacology and therapeutics. 2012;50(11):805-13.

63. Koh KK, Quon MJ, Shin K-C, Lim S, Lee Y, Sakuma I, et al. Significant differential effects of omega-3 fatty acids and fenofibrate in patients with hypertriglyceridemia. Atherosclerosis. 2012;220(2):537-44.

64. Hong XZ, Li LD, Wu LM. Effects of fenofibrate and xuezhikang on high-fat diet-induced non-alcoholic fatty liver disease. Clinical and Experimental Pharmacology and Physiology. 2007;34(1-2):27-35.

65. Belfort R, Berria R, Cornell J, Cusi K. Fenofibrate reduces systemic inflammation markers independent of its effects on lipid and glucose metabolism in patients with the metabolic syndrome. The Journal of Clinical Endocrinology \& Metabolism. 2010;95(2):829-36.

66. Ye P, Li J-J, Su G, Zhang C. Effects of fenofibrate on inflammatory cytokines and blood pressure in patients with hypertriglyceridemia. Clinica chimica acta. 2005;1(356):229-32.

67. Okopien B, Krysiak R, Kowalski J, Madej A, Belowski D, Zielinski $M$, et al. Monocyte release of tumor necrosis factor- $\alpha$ and interleukin- $1 \beta$ in primary type IIa and IIb dyslipidemic patients treated with statins or fibrates. Journal of cardiovascular pharmacology. 2005;46(3):377-86.

68. Okopien B, Krysiak R, Herman ZS. Effects of short-term fenofibrate treatment on circulating markers of inflammation and hemostasis in patients with impaired glucose tolerance. The Journal of Clinical Endocrinology \& Metabolism. 2006;91(5):1770-8.

69. Lalloyer F, Wouters K, Baron M, Caron S, Vallez E, Vanhoutte J, et al. Peroxisome proliferator-activated receptor- $\alpha$ gene level differently affects lipid metabolism and inflammation in apolipoprotein E2 knock-in mice. Arteriosclerosis, thrombosis, and vascular biology. 2011;31(7):1573-9.

70. Shiri-Sverdlov R, Wouters K, van Gorp PJ, Gijbels MJ, Noel B, Buffat L, et al. Early diet-induced non-alcoholic steatohepatitis in APOE2 knock-in mice and its prevention by fibrates. Journal of hepatology. 2006;44(4):732-41.
71. Fathy M, Okabe M, Saad Eldien HM, Yoshida T. AT-MSCs Antifibrotic Activity is Improved by Eugenol through Modulation of TGF- $\beta /$ Smad Signaling Pathway in Rats. Molecules. 2020;25(2):348

DOI: $10.3390 /$ molecules25020348.

72. Niu L, Cui X, Qi Y, Xie D, Wu Q, Chen X, et al. Involvement of TGF- $\beta 1 / \mathrm{Smad} 3$ signaling in carbon tetrachloride-induced acute liver injury in mice. PLoS One. 25;11(5):e0156090.

DOI: 10.1371/journal.pone.0156090.

73. Hagiwara S, Otsuka T, Yamazaki Y, Kosone T, Sohara N, Ichikawa T, et al. Overexpression of NK2 promotes liver fibrosis in carbon tetrachloride-induced chronic liver injury. Liver International. 2008;28(1):126-31.

74. Larsson J, Karlsson S. The role of Smad signaling in hematopoiesis. Oncogene. 2005;24(37):5676-92.

75. Medeiros AI, Sá-Nunes A, Soares EG, Peres CM, Silva CL, Faccioli LH. Blockade of endogenous leukotrienes exacerbates pulmonary histoplasmosis. Infection and immunity. 2004;72(3):1637-1644.

76. Masszi A, Kapus A. Smaddening complexity: the role of Smad3 in epithelial-myofibroblast transition. Cells Tissues Organs. 2011;193(1-2):41-52.

77. Matsumoto K, Morishita R, Moriguchi A, Tomita N, Yo Y, Nishii T, et al. Prevention of renal damage by angiotensin II blockade, accompanied by increased renal hepatocyte growth factor in experimental hypertensive rats. Hypertension. 1999;34(2):279-84.

78. Li J, Liu X, Sha M, Li Y. The balance between HGF and TGF- $\beta 1$ acts as a switch in the tissue remodeling of chronic rhinosinusitis. International Journal of Clinical and Experimental Pathology. 2019;12(3):933-944.

79. Bae EH, Kim IJ, Joo SY, Kim EY, Choi JS, Kim CS, et al. Renoprotective effects of the direct renin inhibitor aliskiren on gentamicin-induced nephrotoxicity in rats. Journal of the Renin-Angiotensin-Aldosterone System. 2014;15(4):348-61.

80. Chanda D, Lee CH, Kim YH, Noh JR, Kim DK, Park JH, et al. Fenofibrate differentially regulates plasminogen activator inhibitor-1 gene expression via adenosine monophosphate-activated 
protein kinase-dependent induction of orphan nuclear receptor small heterodimer partner. Hepatology. 2009;50(3):880-92.

81. Al-Rasheed NM, Al-Rasheed NM, Al-Amin MA, Hasan IH, Al-Ajmi HN, Mohammad RA, et al. Fenofibrate attenuates diabetic nephropathy in experimental diabetic rat's model via suppression of augmented TGF- $\beta$ 1/Smad3 signaling pathway. Archives of physiology and biochemistry. 2016;122(4):186-94.

82. Chen XR, Besson VC, Palmier B, Garcia $\mathrm{Y}$, Plotkine $\mathrm{M}$, Marchand-Leroux $\mathrm{C}$. Neurological recovery-promoting, anti-inflammatory, and anti-oxidative effects afforded by fenofibrate, a PPAR alpha agonist, in traumatic brain injury. Journal of neurotrauma. 2007;24(7):1119-31.

83. Mungunsukh O, Day RM. Transforming growth factor- $\beta 1$ selectively inhibits hepatocyte growth factor expression via a micro-RNA-199-dependent

posttranscriptional mechanism. Molecular biology of the cell. 2013;24(13):2088-97.

84. Harrison P, Bradley L, Bomford A. Mechanism of regulation of $\mathrm{HGF} / \mathrm{SF}$ gene expression in fibroblasts by TGF- $\beta 1$. Biochemical and biophysical research communications. 2000;271(1):203-11. 